\title{
THE EFFECT OF ANTERIOR CRUCIATE LIGAMENT RECONSTRUCTION ON MALE SEXUAL HEALTH
}

\author{
İzzet Bingöl ${ }^{1}$ and Yasin Köker ${ }^{2}$ \\ ${ }^{1}$ Ankara 29 Mayıs State Hospital \\ ${ }^{2}$ Anakara 29 Mayıs State Hospital
}

May 27, 2021

\begin{abstract}
Background: Anterior cruciate ligament (ACL) injuries are among the most common ligament injuries having social, mental, and spiritual effects as well as physical effects, reducing the quality of life. Sexual health plays an important role inreproduction as well as in the physical, intellectual, emotional, and social aspects of life. Aims: In this study, we compare male patients with conservative and surgical treatment after ACL rupture in terms of sexual health. Methods: The sexual health statuses of 41 male patients diagnosed of ACL rupture followed by conservative or surgical treatment were evaluated before treatment, in the 6 months and at the 1 year after treatment and compared using the International Index of Erectile Function-5 (IIEF- 5). Results: When the mean IIEF-5 scores followed with surgery and conservative treatment were compared at 6 months, it was observed that the scores of those receiving surgical treatment were significantly lower $(\mathrm{p}=0.005)$. When the mean IIEF-5 scores at 1 year were compared, it was seen that the surgical treatment scores were significantly higher. $(p=0.012)$. Conclusions: This study has shown that surgical treatment for ACL rupture has more positive effect on male sexual life in the long term compared to conservative treatment.
\end{abstract}

\section{ABSTRACT}

Background: Anterior cruciate ligament (ACL) injuries are among the most common ligament injuries having social, mental, and spiritual effects as well as physical effects, reducing the quality of life. Sexual health plays an important role inreproduction as well as in the physical, intellectual, emotional, and social aspects of life.

Aims: In this study, we compare male patients with conservative and surgical treatment after ACL rupture in terms of sexual health.

Methods: The sexual health statuses of 41 male patients diagnosed of ACL rupture followed by conservative or surgical treatment were evaluated before treatment, in the 6 months and at the 1 year after treatment and compared using the International Index of Erectile Function-5 (IIEF- 5).

Results: When the mean IIEF-5 scores followed with surgery and conservative treatment were compared at 6 months, it was observed that the scores of those receiving surgical treatment were significantly lower (p $=0.005)$. When the mean IIEF-5 scores at 1 year were compared, it was seen that the surgical treatment scores were significantly higher. $(\mathrm{p}=0.012)$.

Conclusions: This study has shown that surgical treatment for ACL rupture has more positive effect on male sexual life in the long term compared to conservative treatment.

Keywords: Anterior cruciate ligament, male sexual health, erectile dysfunction

What's already known about this topic? 
Although sexual health evaluations are made with some surgical techniques and orthopedic surgical techniques, there are no studies on anterior cruciate ligament reconstruction.

\section{What does this article add?}

Surgical technique has been shown to have a positive effect on sexual health in men.

\section{Declarations:}

Funding : This research did not receive any specific grant from funding agencies in the public, commercial, or not-for-profit sectors.

Conflicts of interest/Competing interests : The authors declare that they have no competing interests.

Availability of data and material: This study does not contain any third material.

Code availability: Not applicable

Authors' contributions : Conceptualization; İzzet Bingöl (İ.B.) and Yasin Köker (Y.K.). Investigation; İ.B and Y.K. Writing; I.B. and Y.K.

Ethics approval : The study protocol was approved by the ethics committee 248 of Ankara City Hospital (reference number: E1-20-1386). Informed consent was obtained from all participants.Consent to participate (include appropriate statements)

Consent to participate: All patients signed a written informed consent form before participating in the study.

Consent for publication All the authors have approved the final version and agree to publish

\section{Hosted file}

Manuscript.docx available at https://authorea.com/users/416118/articles/523785-the-effectof-anterior-cruciate-ligament-reconstruction-on-male-sexual-health

\section{Hosted file}

Tables.docx available at https://authorea.com/users/416118/articles/523785-the-effect-ofanterior-cruciate-ligament-reconstruction-on-male-sexual-health 


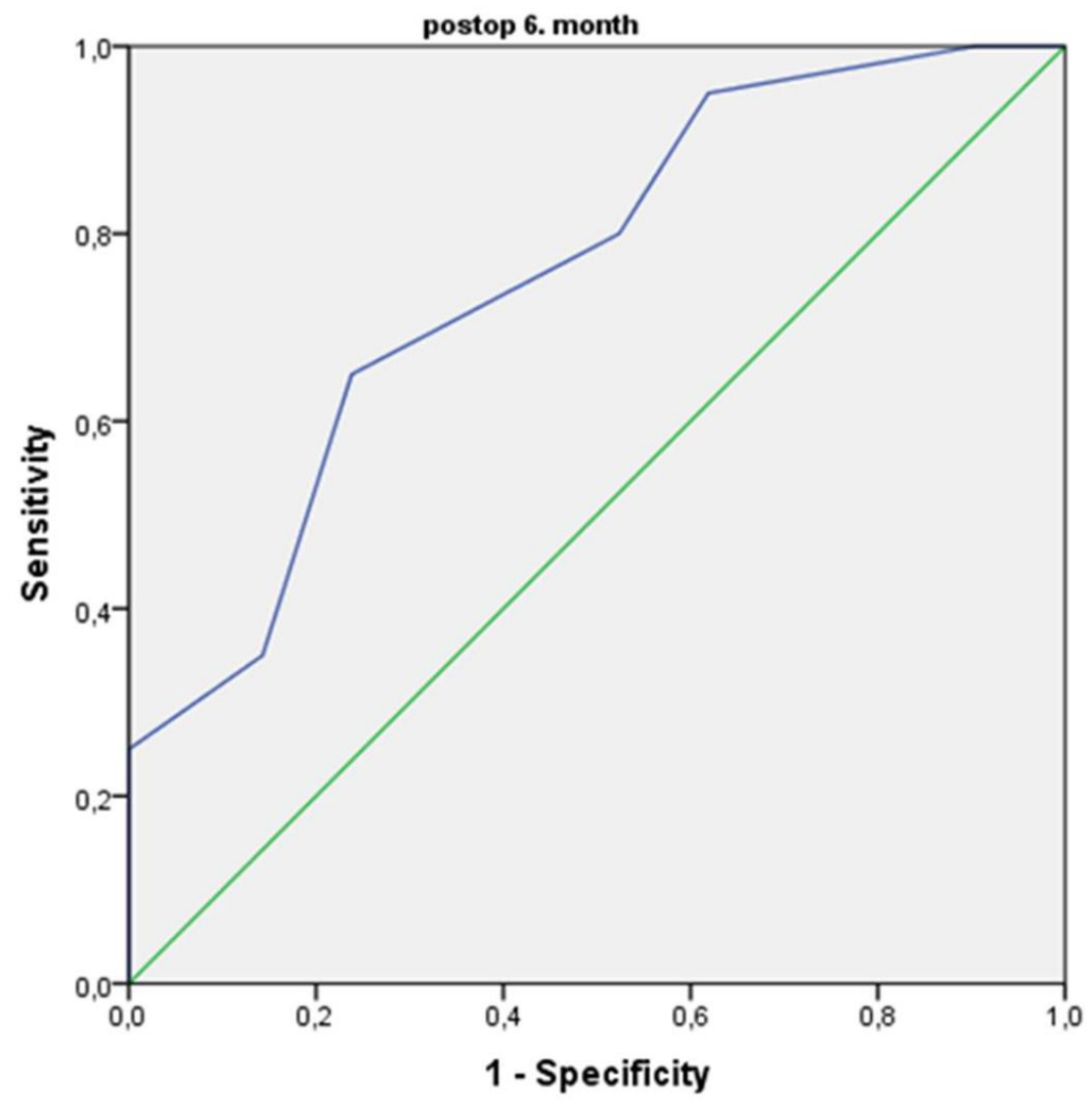




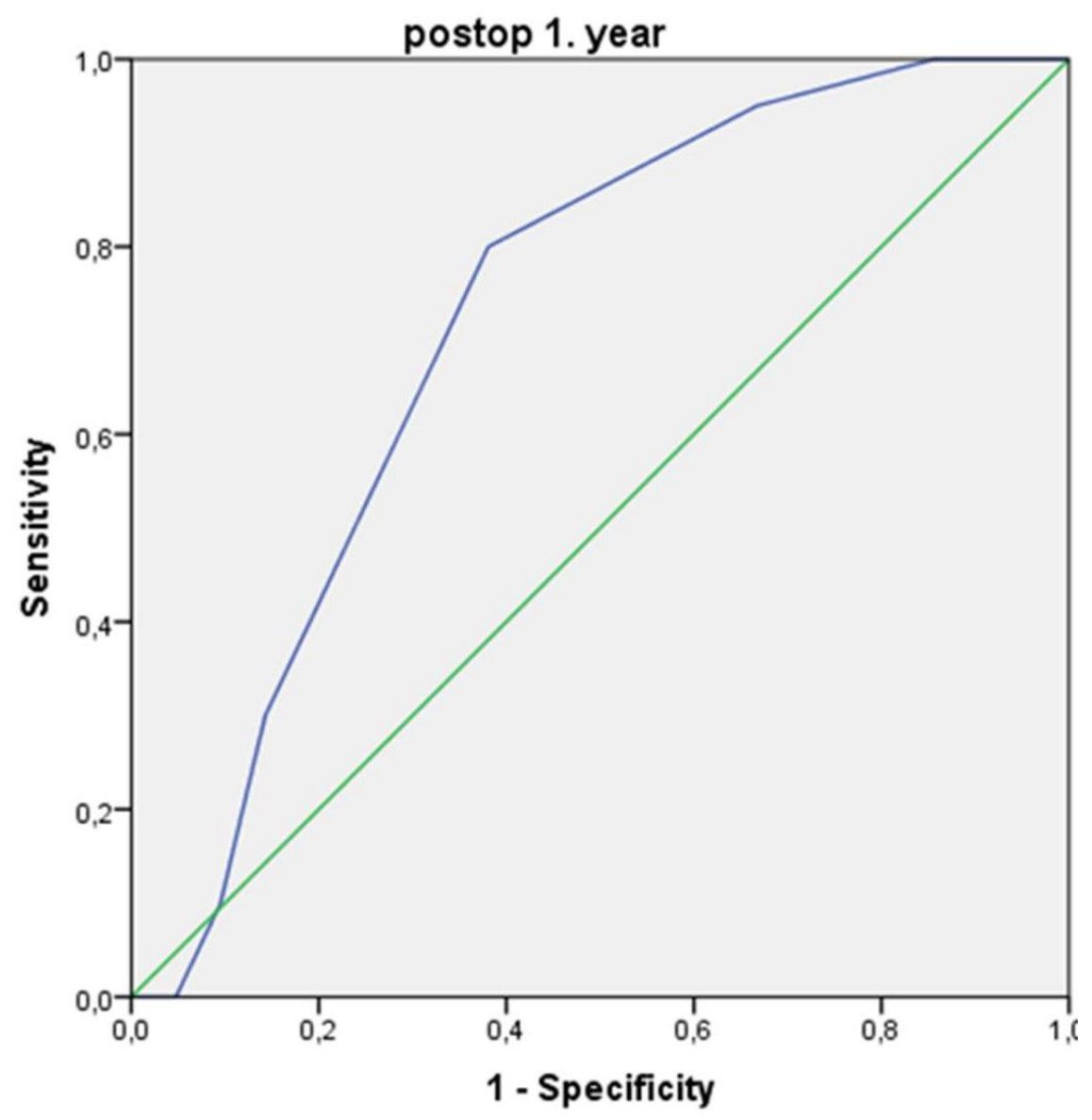

